tained at an adjustable potential, as a function of time after the interruption of a low-voltage are in argon. From a series of such measurements the timevariation of the electron (or ion) concentration and the electron temperature could be determined at various places in the tube. The loss of particles by diffusion to the walls was illustrated by graphs showing the concentration as a function of position within the tube at different times; at first the concentration was practically uniform over the greater part of the tube, but this region of uniformity became more and more closely confined around the axis of the tube as time went on. Within this region, loss by diffusion may be neglected, so that the value of $\alpha$, the coefficient of recombination, may be deduced from the equation $d N$ $\frac{d N}{d t}=-\alpha N^{2}$, where $N$ is the number per eubic centimetre of ions of either sign. Sayers found that $\alpha$ varies with electron temperature, but is independent of the pressure over the range $0 \cdot 1-1 \cdot 0 \mathrm{~mm}$., as would be expected if the recombination is a two-body process. At an electron temperature of $1,000^{\circ} \mathrm{K}$., $\alpha$ for argon is about $6.6 \times 10^{-10} \mathrm{~cm}^{3} \mathrm{sec}^{-1}$.

In comparing these experiments with theory, Prof. Sayers pointed out that the probability of recombination to the ground state of the neutral atom is likely to be only a small fraction of the total recombination probability and, since the excited states will be roughly hydrogen-like for all atoms, it is not unreasonable to compare calculated values of $\alpha$ for oxygen with the experimental values obtained for argon, mercury and cæsium. The variation of $\alpha$ with electron temperature is as predicted by theory, but the absolute magnitude of $\alpha$ is about five hundred times larger than theory predicts. Observations on recombination in the $F$ region of the ionosphere agree with the laboratory experiments, but considerations of the balance of electron population in interstellar space agree with the theoretical value. In contrast to these unresolved discrepancies concerning the cap. ture of electrons into the whole series of states of the atom, measurements by optical methods of the radiative capture of electrons into individual atomic states seem to agree well with theory.

The discussion which followed centred largely around the question of whether, in the laboratory experiments, it is proper to consider the electrons as being in temperature equilibrium at each instant during the few milliseconds occupied by the decay of the ionization.

In the last paper of the afternoon session, Dr. Hoyle discussed one of the most fascinating problems of astrophysics-the process of synthesizing heavy elements from hydrogen and helium, and obtaining the observed distribution of these elements throughout space. There are two main problems to be considered : first, it is necessary to establish the conditions in which this synthesis can take place, and secondly to determine where, in the universe, these conditions are actually to be encountered. Having considered the very great difficulties involved in any theory which envisages conditions other than those of thermal equilibrium between the different elements, Dr. Hoyle proceeded to discuss the results obtained by applying statistical mechanies to the evolution of the heavy elements. He pointed out that from this point of view nuclear reactions can be treated by applying the well-known properties of statistical assemblies of dissociating gases, together with an equation determining the neutron-to-proton ratio as a function of density. This latter equation shows that both the concentration of free neutrons and the ratio of neutrons to protons increase with increasing density, and that to obtain a proportion of heavy elements in accordance with observation, densities of the order of $10^{2} \mathrm{gm}$. per cm. ${ }^{3}$ are required. It can be shown also that to establish equilibrium between the various elements in a time sensibly less than the age of the universe, the region considered must have a temperature of the order of $10^{\circ}$ degrees. The central temperatures of normal stars are too low by a factor of about 100, but in the case of those which have collapsed to a small fraction of their initial radius owing to the exhaustion of their hydrogen supply, temperatures of the required magnitude may be encountered. At some stage during the collapse, depending on the angular momentum, which differs widely from one star to another, the rotating spheroid becomes unstable, and material is thrown off into space from its equator. Now the nuclear reactions which would have to take place in order to reestablish equilibrium at the lower temperatures and densities require the emission of energy, so cooling is rapidly accelerated. Dr. Hoyle suggested that a small fraction of the heavy elements formed in a collapsed star remain 'frozen'. Thus, since the amount of material thrown off may amount to 99 per cent of the total mass of the star, such elements may be present both in the material thrown off and in the residual core. Dr. Hoyle connected the super-nova catastrophe with physical conditions inferred from the hypothesis that the Crab nebula represents the remains of a past super-nova.

Among difficulties raised in the course of the discussion was that of accounting for the presence of heavy elements in all known stars, which contain also hydrogen and helium.

\section{THE GIACOBINID METEOR SHOWER, 1946}

GEVERAL papers describing British observations of the Giacobinid meteor shower, 1946, were presented at a meeting of the Royal Astronomical Society on December 13, 1946. Special interest was attached to this subject because of the incidence of a shower of such exceptional intensity at a time when the considerable advances made by radar techniques were able to make an important contribution to the observations. Such methods not only enabled estimates of hourly rates to be made without interference due to poor visibility, but also demonstrated how information on certain meteor characteristies can be provided more conveniently and more precisely than is possible by visual meteor watches.

An introductory paper on the visual watch was given by J. P. M. Prentice, director of the Meteor Section of the British Astronomical Association. Although observations were considerably hindered by bad weather, it was possible to obtain a complete curve showing the variation in hourly rate by combining the results of several observers. The shower was of approximately six hours duration, rising steeply to a sharp maximum around $0345 \mathrm{hr}$., followed by a steep decline. The variation in rates was so rapid that the mean hourly rates, determined by averaging over short periods, are considerably affected by the length of period chosen. The visual observa. tions were averaged over 7-8 minutes, and the maximum hourly rate corrected to a radiant in the 
zenith was 2250. An allowance for the strong moonlight which prevailed was made by comparing the magnitude distribution with the corresponding distribution during the great shower of 1933, for which the observations of $\mathbf{F}$. de Roy are available. Even when this factor is taken into account, the 1933 shower appeared to be at least twice as rich at all stages in the development as that of 1946. It had been anticipated that the shower would be richer in 1946 since the comet was so much nearer the earth at its node than in 1933, the distances being 135,000 miles in 1946 as compared with 500,000 miles in 1933 .

Dr. A. C. B. Lovell then described the radar observations which he made with C. J. Banwell and J. A. Clegg at the University of Manchester. The aerial system, which was adjustable in both azimuth and elevation, provided a narrow radio beam at a wave-length of $4.2 \mathrm{~m}$. An almost continuous watch was maintained for 71 hours between October 8 and 11. The radio echoes were generally recorded by visual observation of the cathode ray tube; but, in addition, a ciné-film was taken at 8 frames per second during the peak period.

Throughout most of the watch the radio beam was directed at $90^{\circ}$ from the Giacobinid radiant. To confirm that echoes are generally only obtained when the beam is perpendicular to the ionized trails produced by the passage of the meteors, the beam was directed towards the radiant for part of the shower, when it was found that the number of echoes fell to 4 per cent of the previous value.

Observations of the hourly rate of occurrence of echoes were made with the directional aerial system, supplemented by a dipole aerial system giving an almost all-round looking beam. Until $2100 \mathrm{hr}$. U.T. on October 9 , the rate was very low, usually 2 per hour and never greater than 6 per hour. A very slight increase was recorded after $2100 \mathrm{hr}$. U.T. ; but it was not until after $0000 \mathrm{hr}$. on October 10 that the shower suddenly developed. The maximum which occurred between 0340 to $0343 \mathrm{hr}$. U.T. was extremely sharp, and a peak rate of 168 echoes per minute, averaged over a period of 24 sec., was attained. Within $\pm 5 \mathrm{~min}$. of this peak the rate was down to 50 per minute, and within $\pm 30 \mathrm{~min}$. it was 1 per minute. By $0600 \mathrm{hr}$. the shower had passed completely.

An examination of the life histories of echoes recorded on the cine-film showed, in a number of cases, very rapid fluctuations in amplitude with a fairly regular decay superimposed. An analysis of the distributions of heights, durations and amplitudes gave evidence of change during the progress of the shower, thus indicating either variation in the characteristics of the stream or in the conditions of the upper atmosphere. The ciné-film record obtained at the maximum of the shower provided a sufficiently accurate distribution of echo amplitude for an estim. ate to be made of the size distribution of the meteors by means of the theories of meteor ionization de. veloped at the University of Manchester by Herlofson. It was shown that the number of electrons produced per $\mathrm{cm}$. path by the meteor could be determined from the received echo strength, for a radio beam directed at right angles to the meteor train, from the relation received echo power $=8.88 \times 10^{-28} \frac{\alpha^{2} \lambda^{3}}{R^{3}} P_{0} G^{2}$ watts, where $\alpha$ is the number of electrons per cm. path, $R$ the range of the echo in cm., $\lambda$ the wave-length in cm., $P_{0}$ the peak transmitter power in watts, and

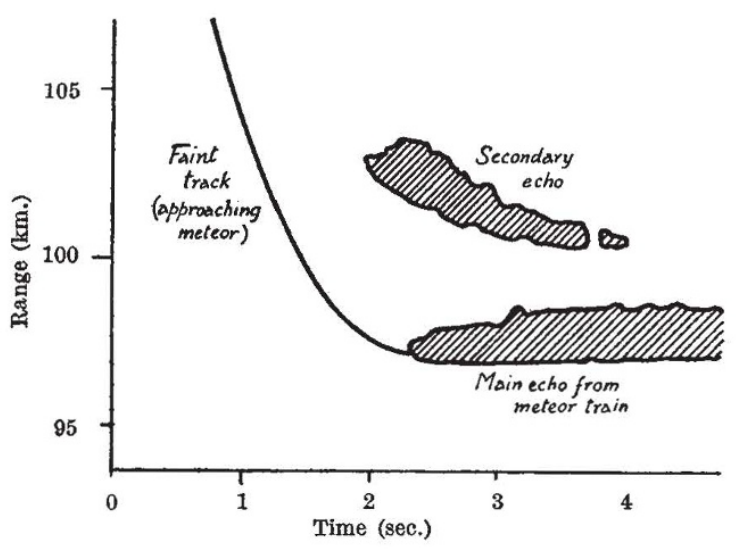

$G$ the power gain of the aerial system (the same for transmitter and receiver) as compared with a halfwave dipole. Calculations by Herlofson showed that $\alpha$ is proportional to the size of the meteor and inversely proportional to the velocity. It was hence deduced from echo-strength measurements during the maximum of the Giacobinid shower that the number of meteors of a given size was inversely proportional to the size.

A visual watch was carried out simultaneously with the radar observation between 0014 and $0107 \mathrm{hr}$. U.T. and 0134 and $0228 \mathrm{hr}$. U.T., when the sky was clear ; 26 per cent of the radio echoes observed during these periods were coincident with visual meteors. If the analysis is restricted to radio echoes of more than 0.5 sec. duration, then 50 per cent of these were coincident with visual meteors. This simultan. eous watch afforded an opportunity of testing Herlof. son's theory, according to which a meteor which produces $10^{10}$ electrons $/ \mathrm{cm}$. path will be at the limit of naked eye visibility. This value was found to be in general agreement with that deduced from calculations of the ionization necessary to give the observed radio echoes.

J. S. Hey gave an account of observations made in collaboration with S. J. Parsons and G. S. Stewart, at the Operational Research Group, Ministry of Supply. A continuous watch was maintained during October 7-11, with radar equipment of about 5 metres wave-length, using both visual and photographic recording of the cathode ray tube display. One of the most important results, which emerged as a consequence of improved photographic methods giving better resolution than hitherto, was the recording of the fine structure of the echoes and the determination of meteor velocity. The echoed signals were made to appear as bright spots on a linear-range trace on the cathode ray tube. This was photographed on a film moving at right angles to the trace at uniform speed. The resulting record thus gave a plot of echo-range against time. Satisfactory resolution was obtained by displaying a limited range-band of $80-115 \mathrm{~km}$. over the full width of the cathode ray tube and by using a $35-\mathrm{mm}$. film moving at $2.4 \mathrm{~mm}$. per sec.

In earlier work by Hey and Stewart (Nature, 158, $481 ; 1946)$, it was shown that the main echoes are obtained at right angles to the meteor trails. Some of the recordings in the present series showed clearly that prior to the formation of the main echo there is a faint fast-moving echo which can be associated with the ionization in the immediate vicinity of the 
approaching meteor. Not until the ionized column approximately reaches the point of intersection of the normal from the observing station to the ionized column can the stronger and more enduring broadside echo be obtained. Secondary reflexions which appeared in the recordings can be attributed t.o the effects of drifts and diffusion which, as is known from visual meteor data, may cause irregularities in the train a few seconds after its formation. An example of a Giacobinid meteor echo, producing sufficient ionization around it for the track of the approaching meteor to be detected, is illustrated in the accompanying diagram, which is taken from a photographic recording.

These faint tracks resulting from the reflexion from the ionization in close proximity to the approaching meteor enabled the geocentric velocities to be determined without requiring a knowledge of the radiant position. The range-time characteristics, with few exceptions, were found to be representative of a body moving with uniform velocity in a straight line. The range $R$ at any instant $T$ is then given by

$$
R^{2}=R_{0}^{2}+V^{2}\left(T-T_{0}\right)^{2}
$$

where $R_{0}$ and $T_{0}$ represent the range and time when the range is a minimum and $V$ is the geocentric velocity. From the analysis of twenty-two tracks, the weighted mean of the computed velocities was found to be $22.9 \mathrm{~km}$. $/ \mathrm{sec}$, with root-ruean-square deviation of $1.3 \mathrm{~km} . / \mathrm{sec}$., the weighting factor being made proportional to the duration of the track. This mean value is in close agreement with the theoretical velocity of $23.7 \mathrm{~km}$. $/$ sec. given by Dr. J. G. Porter, director of the Computing Section of the British Astronomical Association.

Sir Edward Appleton and R. Naismith, who in 1932 noticed the general occurrence of the transient jonospheric radio (or radar) echoes which are now known to arise from the ionization trails of meteors, took part in the discussion. The results which they obtained on the occasion of the Giacobinid Shower of October 10 were described in these columns a few weeks ago (Nature, 158, 936; 1946). Moreover, as a result of their systematic study of the diurnal and seasonal variations of both meteor ionization trails and sporadic ionization in the $E$ layer of the irmosphere, they have been led to the important conclusion that the fine dust of sporadic meteors contributes substantially to the irregularities of ionization which have long been known to exist in the $E$ layer. An account of these phenomena, and the descriptions of the investigations of the other radar workers mentioned above and of 60 Group R.A.F., are being given at a meeting of the Physical Society on January 31. J. S. Hwy

\section{NEWS and VIEWS}

\section{Gold Medal of the Royal Astronomical Society:} Prof. M. G. J. Minnaert

Prof. Marcel Gilles Jozef Minnaert, director of the Sonnenburg Observatory, Utrecht, has been awarded the Gold Medal of the Royal Astronomical Society for his outstanding contributions to solar physics and in particular to solar spectrophotometry. A pupil of Julius, he was later at Utrecht a colleague of Ornstein and Moll, and an early worker in the field of spectrophotometry, both developing the technique and applying it to a wide range of solar problems. He has taken part in a number of eclipse expeditions : to Sumatra in 1926 and 1929, and to Cenada in 1932, all spoilt by clouds; his one successful expedition was to Lapland for the eclipse of 1927, where he obtained the first absolute values of the intensities of the chromospheric lines. He produced during the early days of the War a "Photometric Atlas of the Solar Spectrum", a most valuable contribution to solar spectroscopy. His later years in the War were spent in a concentration camp, from which he has emerged with his scientific enthusiasms undamped. $\mathrm{He}$ is chairman of the Commission on Spectrophotometry of the International Astronomical Union, a position for which he is well qualified by both experimental and theoretical studies. The distribution of energy in the sun's continuous spectrum and in that of the corona, the law of darkening of the sun's limb, and the polarization of the corona are among other subjects to which he has made valuable contributions. He has also worked on the direct photometry of Venus, the moon and red stars. His work is throughout characterized by a thoroughness, accuracy and care which have secured him a leading position in his own field. $\mathrm{He}$ was elected an associate of the Royal Astronomical Society in 1945 .

\section{Cadman Medal of the Institute of Petroleum: Mr. R. P. Russell}

THe Council of the Institute of Petroleum has awarded the Cadman Memorial Medal to Mr. Robert Price Russell, president of the Standard Oil Development Co., the central technical and research organisa. tion of the Standard Oil Company (N.J.). Great services were rendered by Mr. Russell and his associates during the War in the production of high. octane aviation fuels, synthetic rubbers and toluene for explosives, and in the development of flame throwers, incendiaries and smoke generators. More than half the American output of aviation petrol for war-planes was manufactured by the catalytic cracking process, in which Mr. Russell played a notable part. Nine tenths of the American output of petroleum-based butadiene, the starting material in the manufacture of synthetic rubber, came from the process which Mr. Russell directed into largescale production. In recognition of his war-time work, Mr. Russell was awarded the Medal for Merit, the highest civilian award in the United States. In 1946 he received the gold medal of the American Institute of Chemists for "noteworthy and outstand. ing service to the science of chemistry". Mr. Russell is well known personally in Great Britain, for he came here in 1944 as chairman of the Petroleum, Chemical and Rubber Division of the U.S. Strategic Bombing Survey. A team of scientific workers under his supervision entered Germany on the heels of the front-line troops to survey the enemy's vast oilchemical industry.

Born in Massachusetts in 1898, Mr. Russell saw military service in the First World War; on resuming his studies, he obtained a master's degree in chemical engineering at the Massachusetts Institute of Technology in 1923. After holding some academic 